\title{
TFIIH (BTF2), à l'interface de trois processus cellulaires: transcription, réparation et cycle cellulaire
}

Continuellement exposé à l'action d'agents mutagènes environnants, comme les cancérogènes chimiques, les radiations ionisantes ou les UV, l'organisme subit au niveau de son ADN de nombreux dommages qu'il doit réparer afin de conserver l'intégrité de son information génétique et d'éviter ainsi un dysfonctionnement des cellules pouvant conduire à leur mort ou à leur transformation en cellules cancéreuses. Dans chaque cellule, la localisation et la réparation d'une lésion doivent intervenir avant la phase $\mathrm{S}$ de réplication de l'ADN pour éviter que l'erreur génétique ne soit transmise aux cellules-filles ; aussi faut-il que le cycle cellulaire soit provisoirement arrêté, puis qu'un signal de fin de réparation autorise à nouveau sa progression et la reprise des processus de réplication et de transcription. Les facteurs moléculaires qui contrôlent étroitement l'imbrication de ces différents phénomènes agissent très probablement en des points stratégiques de convergence de ceux-ci.

Il avait déjà été montré qu'outre son rôle clé dans la transcription des gènes codant pour les protéines - il participe à la formation du complexe de préinitiation (comprenant les facteurs généraux TFIIA, B, D, E et $\mathrm{F}$ et l'ARN polymérase II) sur un promoteur minimum (TATA-box et site d'initiation) - le facteur TFIIH (BTF2) intervenait également dans la réparation de l'ADN (figure 1) [1, 2]. A ce jour, il est en outre le seul des facteurs généraux de transcription connus à posséder diverses activités enzymatiques (Tableau I): (1) deux activités hélicases de sens opposés, probablement impliquées dans la séparation des deux brins d'ADN qui $\mathrm{m} / \mathrm{s} n^{\circ} 6$, vol. 11 , juin 95 permettra la lecture du brin codant au cours de la transcription ou l'excision du fragment lésé suivi de sa resynthèse lors de la réaction de réparation; (2) une activité protéinekinase capable, entre autres, de phosphoryler le domaine carboxy-terminal (CTD pour carboxy-terminal domain) de la grande sous-unité de l'ARN polymérase II (ARNpol II). Les activités hélicases résident dans les sous-unités ERCC2 (XPD) et ERCC3 (XPB), qui sont des composants essentiels de la machinerie de réparation par excision (NER pour nucleotide excision repair). Des mutations dans les gènes ERCC2 et ERCC3 sont responsables d'une déficience sévère de la réparation, le xeroderma pigmentosum (XP), caractérisée par une hyperphotosensibilité et un risque accru de développer des cancers cutanés, associée aux symptômes d'une autre maladie génétique, le syndrome de Cockayne, où le couplage réparation-transcription est aboli $[3,4]$. Certains patients du groupe de complémentation XPD sont atteints, en plus, d'une autre maladie récessive rare, la trichothiodystrophie $[3,4]$. Cette dernière pourrait être également le résultat d'une mutation dans une sous-unité de TFIIH non encore identifiée [5]. On ne connaît pas d'activités enzymatiques liées aux sous-unités p62 et p44 ; cependant, il a été récemment montré que leurs homologues chez Saccharomyces cerevisicue, Tfbl et Ssll, étaient requis aussi bien pour la transcription que pour la réaction NER [6].

L'activité kinase utilisant le CTD comme substrat n'avait pu être jusqu'alors attribuée à aucune sous-unité et son importance dans la fonction de TFIIH reste mal élucidée. Il a été montré en outre que c'est la forme non phosphorylée de l'ARNpol II qui s'incorpore préférentiellement dans le complexe de préinitiation et que la phosphorylation du CTD précède ou est concomitante de l'amorçage de la transcription ; elle permettrait à la polymérase de se libérer de son ancrage à la protéine TBP (TATA-box binding protein) lorsque cette dernière est fixée au promoteur. Bien qu'il ne soit pas prouvé que cette étape soit obligatoire pour la transcription de tous les gènes, on sait que le CTD est toujours phosphorylé lorsque la polymérase est engagée dans le processus d'élongation. Par ailleurs, la conversion du complexe d'initiation en complexe d'élongation nécessite l'hydrolyse d'ATP, vraisemblablement en rapport avec les fonctions hélicase et kinase et/ou un besoin énergétique autre (libération de certains facteurs, déplacement du complexe...).

Dans la stratégie adoptée afin de caractériser les différents composants de TFIIH, nous avons récemment soumis deux polypeptides à une digestion trypsique partielle. Le microséquençage du polypeptide de poids moléculaire $40 \mathrm{kDa}$ a permis d'identifier une protéine préalablement connue pour son rôle dans la régulation du cycle cellulaire: la protéine kinase MO15 [7]. Celle-ci est la sous-unité catalytique d'un complexe protéique responsable de la phosphorylation des protéines p34 cilc2(ctkl) et p33 ${ }^{\alpha / k 2}$, deux kinases dépendantes des cyclines (Cdk) qui contrôlent, respectivement, l'entrée de la cellule en mitose et la transition de la phase Gl vers la phase S [8]. Cette phosphorylation intervient au niveau d'un résidu thréonine qui est conservé 
dans toutes les kinases apparentées à Cdc2 et induit leur activation par un changement conformationnel qui découvre leur site catalytique. Les activités de Cdc2 et Cdk2 sont donc réglées non seulement par l'association à différentes cyclines mais aussi par un mécanisme de phosphorylation/déphosphorylation. Tout comme ses propres substrats dans le cycle cellulaire, MO15 est une kinase dépendante des cyclines: elle est associée à la cycline $\mathrm{H}$, qui s'est avérée être la seconde sous-unité que nous avons microséquencée [7]. Son activité CAK (pour cdk-activating kinase) peut être mesurée in vitro par la phosphorylation du complexe cdk2cycline A ; ce dernier devenu ainsi actif pourra, dans un deuxième temps, phosphoryler l'histone $\mathrm{Hl}$ en présence d'ATP radiomarqué. Ainsi, en constatant, lors d'immunoprécipitations effectuées avec des anticorps spécifiques dirigés contre les différentes sous-unités de TFIIH ou contre la protéine MO15 elle-même, que l'activité CAK était associée aux activités de phosphorylation du CTD et de transcription de TFIIH, l'appar- tenance du couple MOl5/cycline $\mathrm{H}$ au facteur a pu être démontrée, sans que l'on sache toutefois avec quelle(s) sous-unité(s) les deux partenaires sont susceptibles d'interagir ni avec quelle force ils sont liés au complexe protéique de base. Existe-t-il différents complexes TFIIH qui rempliraient des fonctions différentes selon que leur composition en sousunités varie, en particulier suivant la présence ou non de MO15 et de la cycline $\mathrm{H}$ ? Cette hypothèse ne peut être complètement exclue. En effet, l'analyse quantitative des expériences d'immunoprécipitation a révélé des différences importantes suivant la nature de l'anticorps utilisé. En partant d'un extrait brut de cellules HeLa, les anticorps dirigés contre la sous-unité p62 précipitent moins de polypeptides MO15 et ERCC2 que des autres sous-unités du complexe TFIIH ; inversement, les anticorps anti-MO15 ou anti-ERCC2 précipitent chacun MO15 et ERCC2 dans des proportions similaires, et nettement moins de p62. Il semblerait qu’il existe une hétérogénéité dans la composition du complexe TFIIH ; le

Tableau I

LES DIFFÉRENTES SOUS-UNITÉS DU COMPLEXE TFIIH HUMAIN

\begin{tabular}{|c|c|c|c|}
\hline $\begin{array}{l}\text { Masse molaire } \\
\quad(\mathrm{kDa})\end{array}$ & Dénomination & Caractéristiques & $\begin{array}{c}\text { Homologues chez } \\
\text { Saccharomyces } \\
\text { cerevisiae }\end{array}$ \\
\hline $\begin{array}{l}89 \\
80 \\
62 \\
44\end{array}$ & $\begin{array}{c}\text { XPB/ERCC3 } \\
\text { XPD/ERCC2 } \\
\text { p62 } \\
\text { p44 }\end{array}$ & $\begin{array}{l}\text { Hélicase } 3^{\prime}-5^{\prime} \\
\text { Hélicase } 5^{\prime}-3^{\prime} \\
\text { Liaison à l'ADN } \\
\text { (structure en } \\
\text { doigt de zinc) }\end{array}$ & $\begin{array}{c}\operatorname{Rad} 25 / \mathrm{Ss} \mid 2^{*} \\
\text { Rad3 }^{*} \\
\text { Tfb } 1^{*} \\
\text { Ssl 1* }\end{array}$ \\
\hline 41 & p41 & & \\
\hline 40 & MO15 & $\begin{array}{l}\text { Activités CAK et } \\
\text { CTD kinase }\end{array}$ & Kin28 \\
\hline 34 & p34 & $\begin{array}{l}\text { Structure en doigt } \\
\text { de zinc }\end{array}$ & \\
\hline 34 & cycline $\mathrm{H}$ & $\begin{array}{l}\text { Protéine } \\
\text { régulatrice } \\
\text { de } \mathrm{MO15}\end{array}$ & Ccl1 \\
\hline 32 & p32 & $\begin{array}{c}\text { associée au } \\
\text { complexe kinase }\end{array}$ & \\
\hline
\end{tabular}

$X P B, X P D$ : groupe de complémentation $B, D$ du xeroderma pigmentosum.

CTD kinase : carboxy-terminal domain phosphorylating kinase; CAK : pour cdk-activating kinase. 880 fait que MO15 et ERCC2 soient immunoprécipités avec une stœchiométrie constante, indépendamment de l'utilisation d'anti-MOl5 ou d'anti-ERCC2, suggère une interaction préférentielle entre ces deux sous-unités. Ce point est également confirmé par des expériences de "déplacement» (shift) de protéines sur gradient de glycérol où l'on constate un déplacement de la sousunité MO15 parallèlement à celui des activités CAK et CTD-kinase, mais avec seulement une partie des sousunités de TFIIH. Si le complexe TFIIH n'est pas déplacé par un anticorps contre MOl5, il semble néanmoins que la stœchiométrie de sa composition ainsi que ses propriétés en soient altérées; en effet, outre la perte de MO15, près d'un tiers des molécules ERCC2 présentes dans le complexe de départ sont entraînées avec le complexe contenant la kinase, confirmant la possibilité d'une interaction entre ces deux sous-unités. De plus, le déplacement de MO15 provoque une diminution conséquente de l'activité de transcription de TFIIH, vraisemblablement par désassemblage du complexe TFIIH actif.

Jusqu'alors, on supposait que la kinase MO15 n'était impliquée que dans le contrôle du cycle cellulaire [8]. Comme le complexe TFIIH joue un rôle à la fois dans la réparation par excision et dans la transcription $[7,9]$, nous avons voulu montrer que MO15 pouvait également affecter ces deux processus. Pour ce faire, nous avons fait des micro-injections d'anticorps dirigés contre MO15 directement dans des fibroblastes humains, puis nous avons mesuré leur effet sur la transcription, d'une part, et sur la réparation consécutive à une irradiation aux UV, d'autre part, en dosant respectivement l'incorporation d'UTP et de d'ATP radioactifs. Par comparaison avec des cellules non traitées ou ayant reçu des anticorps contrôle par micro-injection, nous avons pu constater une réduction radicale des deux activités testées. Il restera cependant à déterminer si MO15 intervient de façon directe dans les processus de réparation et de transcription en modifiant l'un ou l'autre de ses partenaires comme 


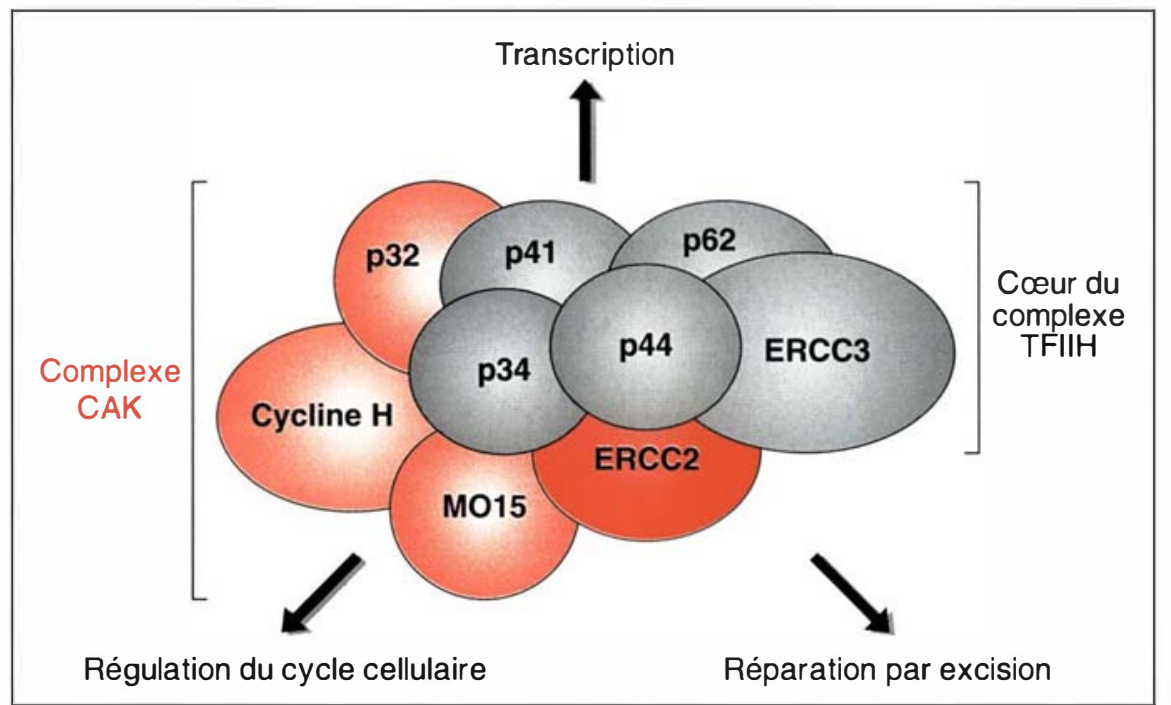

Figure 1. Sous-unités du complexe de transcription TFIIH. $p 32$, la cycline $H$ et MO15 forment le complexe CAK (pour cdk-activating kinase) qui est au cœur de la régulation du cycle cellulaire. La sous-unité ERCC2 (en rouge) pourrait faire la liaison entre ce complexe et le cœur du complexe TFIIH (en gris). ERCC2 et ERCC3 sont impliquées dans la réparation par excision de nucléotides. De plus, il a été démontré que les homologues de levure de p44 (Ss/1) et p62 (Tfb1) sont impliqués dans la résistance aux UV.

l'ARNpol II ou certains facteurs de réparation et de transcription. Le complexe kinase pourrait également intervenir indirectement comme composant structural du complexe TFIIH. Des éléments de réponse concernant les interactions entre les différentes sous-unités de TFIIH pourront être apportés par des expériences de coexpression des différentes sous-unités dans des cellules d'insecte infectées par des baculovirus recombinants ainsi qu'au moyen du système double-hybride chez la levure $S$. cerevisiae.

L'importance de l'interaction entre MO15 et ERCC:2 reste encore à déterminer, mais il est d'ores et déjà possible d'imaginer qu'elle puisse faire office de point de régulation favorisant, suivant le cas, la progression du cycle cellulaire, la transcription ou la réparation du génome après son endommagement. Des études parallèles effectuées sur le facteur TFIIH de $S$. cererisiae confirment les résultats que nous avons obtenus chez l'homme en identifiant Kin28 et la cycline Ccll comme les homologues respectifs, dans cet organisme, $\mathrm{m} / \mathrm{s} n^{\circ} 6$, vol 11 , juin 95 et surtout par la phosphorylation d'un résidu thréonine bien défini. Une mutation de ce site de phosphorylation abolit l'activité CAK, tandis que MO15 surexprimée che\% $E$. coli, et donc sans modification post-traductionnelle, n'acquiert cette activité qu'après incubation avec un extrait de cellules eucaryotes.

Jusqu'à présent, aucun facteur modulant l'activité CAK n'a pu être identifié puisque la cycline $\mathrm{H}$ semble toujours associée à MOl5 et qu'on ne connaît pas encore la ou les kinases phosphorylant MO15. Chez la levure, un autre couple kinase/cycline, SRB10/SRB 11, appartenant à l'holoenzyme ARNpol II et impliqué dans la régulation de la transcription in vivo, est capable de phosphoryler le CTD in vitro [11 ]. Cette activité pourrait, parallèlement à l'activation d'autres kinases par SRB10/SRB11, s'ajouter à l'activité de phosphorylation de MO15 lors d'une activation de la transcription. De même, on ne peut exclure la possibilité que ERCC2 puisse également affecter l'activité de MO15. En effet, on soupçonne cette hélicase de jouer un rôle dans la reconnaissance des lésions de l'ADN, rôle que tient $\operatorname{Rad} 3$, son homologue chez S. cerevisiae [2]. Cette reconnaissance pourrait entraîner une modulation de l'activité CAK de MO15 via son association à ERCC2, qui fait peut-être suite à un changement conformationnel ; la kinase pourrait alors phosphoryler des protéines du cycle cellulaire plus en aval. Cela rejoindrait l'hypothèse selon laquelle le complexe d'élongation des ARN messagers aurait un rôle de surveillance de l'intégrité des régions transcrites: son blocage par une lésion engendrerait un signal d'arrêt du cycle cellulaire et de recrutement de la machinerie de réparation qui se substituerait à celle de transcription. Des résultats obtenus en collaboration avec d'autres laboratoires font état de la liaison de la protéine suppresseur de tumeur p53 au facteur TFIIH ([12] ; T. Léveillard, communication personnelle). Le rôle régulateur de p53 dans le cycle cellulaire à la suite d'un endommagement de l'ADN lui a valu le titre de "gardien du génome "; en effet, après une irradiation, la quantité de p53 aug- 
mente dans la cellule et la protéine stimule la transcription de différentes protéines impliquées dans le cycle cellulaire, le processus de réparation et l'apoptose [13]. La région C-terminale de p53 se lie spécifiquement à la partie $\mathrm{N}$-terminale de ERC'C2, a u niveau du site de liaison de l'ATP, ainsi qu'au domaine hélicase III de ERCC.3. Il en résulte une inhibition des activités hélicases, plus forte pour ERCC.2 que pour ERC.C3. La protéine p53 interagit également avec p62. Elle pourrait ainsi contrôler la réparation couplée à la transcription via une modulation des activités hélicases; une altération de cette régulation pourrait être une étape vers la transformation cellulaire. En effet, différents mutants de p53, mis en évidence dans certaines tumeurs, lient plus fortement ERCC.2 que ne le fait p53 normale et pourraient séquestrer l'hélicase ou modifier son action.

Si l'existence de plusieurs complexes TFIIH se voit confirmée, leur composition en sous-unités ainsi que l'identification de leurs partenaires respectifs devrait nous permettre de mieux définir leur(s) rôle(s) dans chacun des trois processus que sont la transcription, la réparation et le cycle cellulaire. Avec la mise en évidence du complexe kinase/cycline $\mathrm{H}$, après celle des activités hélicases et ATPase dépendante de l'ADN, on commence à envisager quels seront les points de contrôle et donc de régulation des différents mécanismes orchestrant le bon déroulement du cycle cellulaire

\section{Richard Roy \\ Etienne Bergmann Jean-Marc Egly}

Institut de génétique et de biologie molécutlaire et cellulaire (Cnrs/Inserm), Université Louis-Pasteur de Strasbourg, 1, me Laurent-Fries, BP 163, 67404 Illkirch. Cedex, France.

\section{TIRÉS À PART}

\section{RÉFÉRENCES}

1. Schaeffer L, Egly J. BTF2/TFIIH, un facteur de transcription et réparation implique dans des maladies de la réparation de l'ADN. médecine/sciences 1994; 10: 973-8.

2. Seroz T, Hwang JR, Moncollin V, Egly JM. TFIIH: a link between transcription, DNA repair and cell cycle regulation. Curr $O p$ Gen Dew 1995; 5 : 217-21.

3. Sarasin A. La réparation de l'ADN au centre de la biologie de la cellule. médecine/sciences $1994 ; 10$ : 951-2.

4. Sarasin A. Les gènes humains de la réparation de l'ADN. médecine/sciences 1994; 10 43-54

5. Vermeulen W, van Vuuren AJ, Appeldorn E, Weeda (G, Jaspers NGJ, Bootsma D, Lehmann AR, Chipoulet M, Schaeffer L, Egly JM, Hoeijmakers JHJ. Three excision repair proteins in transcription factor a transcription syndrome. Cold Spring Harbor Symposia Serie 1994, sous presse.

6. Wang Z, Buratowski S, Svejstrup JQ, Feaver WJ, Henry NL, Wu X, Komberg R Donahue TF Friedberg EC. The veast TFB1 and SSL1 genes which encodes subunits of transcription factor TFIIH, are required for nucleotide excision repair and RNA polymerase II transcription. Mol Cell Biol 1995 15: 2288-93.

7. Roy R, Adamczewski JP, Seroz T, Vermeulen W, Tassan JP, Schaeffer L, Nigg EA, Hoeijmakers JHJ, Egly JM. MO15 kinase involved in cell cycle regulation is part of TFIIH the transcription/DNA repair factor. Cell 1994; 79 : 1093-101.

8. Darbon J, Fesquet D, Cavadore J. De nouveaux régulateurs du cycle cellulaire: les protéines modulatrices des complexes Cdkcyclines. médecine/sciences 1995; 11 : 349-56.

9. Aboussekra A, Biggerstaff M, Shivji M, Vilpo J. Moncollin V, Podust V, Protic M, Hubsher U, Egly JM, Wood RD. Mammalian DNA nucleotide excision repair reconstituted with purified protein components. Cell $1995 ; 80: 659-68$.

10. Feaver WJ, Svejstrup JQ, Henry NL, Komberg $R$. Relationship of CDK-activating kinase and RNA polymerase II CTD kinase TFIIH/TFIIK. Cell 1994: 79: 1103-9.

11. Liao SM, Zhang J, Jeffery DA, Koleske AI, Thompson CM, Chao DM, Viljoen M van Vuuren HIJ, Young RA. A kinase-cyclin pair in the RNA polymerase II holoenzyme. Nature 1995; 374 : 193-6.

12. Wang $X W$, Yeh H, Schaeffer L, Roy R, Moncollin V, Egly JM, Wang Z, Friedberg EC, Evans MK, Bohr VA, Weeda G, Hoeijmakers JHJ, Forrester K. Harris C.C. p5 modulation of BTF? /TFIIH associated nucleotide excision repair activity. Nature Genel 1995, sous presse.

13. Kahn A. Cycle cellulaire, cancer, sénescence et p53. médecine/sciences $1994 ; 10$ : $206-7$. 